\title{
Management of psoriatic patients in biologic treatment associated with infectious comorbidities
}

\author{
Bernardini Nicoletta ${ }^{1}$, Narcisi Alessandra ${ }^{2,3}$, Skroza Nevena $^{1}$, Ersilia Tolino ${ }^{1}$, Daniela Colapietra ${ }^{1}$, Mastroianni Claudio ${ }^{4}$, \\ Potenza Concetta ${ }^{1}$
}

\begin{abstract}
'Dermatology Unit “Daniele Innocenzi”, Department of MEDICO-Surgical Sciences and Bio-Technologies, Sapienza University of Rome, Fiorini Hospital, Polo Pontino, Terracina, Italy

2Dermatology Unit, Department of Biomedical Sciences, Humanitas University, Rozzano-Milan, Italy

${ }^{3}$ Humanitas Clinical and Research Centre, Skin Pathology Lab, Rozzano-Milan, Italy

${ }^{4}$ Department of Public Health and Infectious Diseases, Azienda Ospedaliera Universitaria Policlinico Umberto I, Sapienza University of Rome, Italy
\end{abstract}

Adv Dermatol Allergol 2020; XXXVII (3): 417-421

DOI: https://doi.org/10.5114/ada.2020.96155

\begin{abstract}
Introduction: Psoriasis is a chronic inflammatory disease affecting about $2 \%$ of population, involving both acquired and innate immunity. Psoriasis affects mainly skin, presenting multiple co-morbidities; among them infective ones. Re-activation of tuberculosis or viral hepatitis (HBV and HCV) still represents a therapeutic challenge in patients receiving treatment with biological drugs, as well as HIV infection. For this reason, a multidisciplinary approach with global treatment resulting from active collaboration of different specialists is highly recommended.

Aim: To investigate the most common infective diseases as co-morbidities associated with psoriasis and to provide algorithms for screening, follow-up and therapeutic management in psoriatic patients.

Material and methods: We examined the main infectious comorbidities that can affect moderate to severe psoriatic patients, influencing the therapeutic choice as during the biological treatment both viral and tuberculosis re-activation may occur. We have therefore evaluated the main diseases (TB, Hepatitis B and C, HIV) and the monitoring of patients during treatment with biological agents.

Results: Regular monitoring of psoriatic patients is recommended during long-term treatment with biological drugs in order to identify cases of re-activation of the latent infective agent or de novo acquired infection.

Conclusions: Here we report the state of art regarding management of psoriatic patients with these co-morbidities suggesting a specific screening and management for infectious diseases in patients with moderate to severe plaque psoriasis.
\end{abstract}

Key words: psoriasis, management, tuberculosis, infectious diseases.

\section{Introduction}

Psoriasis is an inflammatory chronic complex disease which involves both acquired and innate immunity [1]. It affects about $2 \%$ of the Caucasian population, and considering the gender, men and women are equally affected [2]. Psoriasis is associated with multiple comorbidities, for this reason a multidisciplinary approach is highly recommended [3]. Novel therapeutic strategies and the tailoring of treatment itself according to clinical or genetic biomarkers are the goals to be achieved in the future [3]. It is a heterogeneous disorder with many different phenotypes; above all plaque psoriasis is the most frequent one, accounting for $80-90 \%$ of cases [1]. About $50 \%$ of patients show nail involvement [4]. Joint disease is reported in up to $30 \%$ of cases: following cutaneous involvement in 75\% of cases, preceding it in 10\% of cases, or presenting concurrently in the remaining $15 \%$ [5]. A severe form of psoriasis, the involvement of perianal/intergluteal folds as well as the umbilical region, the scalp, and nails represent predictive factors for joint involvement [6]. Many infectious comorbidities have been reported in psoriatic patients, such as latent tuberculosis infection (LTBI) [7], viral hepatitis (HBV and HCV) [8] and HIV infection [9].

Address for correspondence: Bernardini Nicoletta, Dermatology Unit “Daniele Innocenzi”, Department of MEDICO-Surgical Sciences and Bio-Technologies, Sapienza University of Rome, A. Fiorini Hospital, via Firenze, snc, Polo Pontino, 04019 Terracina, Italy, phone: +390773 708531, fax: +390773 708399, e-mail: nicoletta.bernardini@libero.it Received: 18.05.2018, accepted: 17.11.2018 


\section{Aim}

Herein we report an overview on the main infectious disease and the therapeutic management of psoriatic patients with those comorbidities.

\section{Material and methods and results}

Infective diseases could be considered possible comorbidities associated to psoriasis. We examined the main infectious comorbidities that can affect psoriatic patients who need systemic therapy. We have evaluated the main diseases (TB, hepatitis B and C, HIV) and the monitoring of patients during treatment with biological agents.

\section{Tuberculosis (TB)}

Tuberculosis (TB) is caused by Mycobacterium tuberculosis through the respiratory tract in case of pulmonary involvement [10]. Active TB is a contraindication to biological treatment, while latent TB requires treatment with Isoniazid $300 \mathrm{mg} /$ daily + vitamin $B_{6}$ to be started 1 month before the onset of therapy with biological drugs. This treatment should be continued for 6 months given the high risk of re-activation of a latent TB in patients treated with biologics. Thus, all national and international guidelines recommend evaluating the presence of active and latent TB in the patients that will be treated with biological drugs [11-14].

\section{Monitoring of patients during treatment with biological agents}

Regular monitoring of psoriatic patients is recommended during long-term treatment with biological drugs to identify cases of re-activation of latent TB or de novo acquired tuberculosis infection [14-16]. All the patients should be carefully evaluated in the case of persistent cough (for at least 3 weeks), fever, chest pain, haemoptysis, weakness or fatigue, weight loss, night sweating and extra-pulmonary symptoms. If the onset of an active TB occurs during biologic agent administration, treatment must be discontinued and anti-tubercular treatment immediately started.

Retreatment with biological therapy should be considered case by case [17]. On the basis of clinical judgment, IGRA test (Interferon-Gamma Release Assay) could be performed every 12 months, and in case of positive results during treatment with tumor necrosis factor $\alpha$ (TNF- $\alpha$ ), interleukins (IL)-12/23 or IL-17 blockers, it is necessary to consult an infectious diseases physician $[18,19]$.

\section{HBV infection}

HBV infection affects one-third of the population worldwide and can be complicated by cirrhosis and hepatocellular carcinoma (HCC) $[20,21]$. Re-activation of
HBV is a well-known phenomenon in chronic HBV carriers, it can occur in patients undergoing immunosuppressive therapy, such as those receiving biological drugs for the treatment of psoriasis [22, 23]. Thus, it is crucial to perform an accurate screening in all patients requiring a biologic drug. Vaccination is recommended for all patients before starting biological therapies [24].

\section{Monitoring of patients during treatment with biological agents}

Occult HBV carriers may undergo biologic treatment following a strict clinical/laboratory follow-up procedure. In such cases, HBV-DNA and HBsAg should be monitored every 6 months; if HBV-DNA is detectable, antiviral treatment should be started.

In HBV inactive carriers ( $\mathrm{HBSAg}+$ ), HBV-DNA and quantitative HBsAg should be monitored. If HBV-DNA is detectable, treatment with entecavir or tenofovir should be started before the biologic drug. If HBV-DNA is not detectable, antiviral prophylaxis with lamivudine is appropriate and should be started 2 weeks before the start of biologic treatment and continued up to 6 months after the end of treatment. HBV-DNA and liver function tests should be monitored every 6 months and an ID consultation is required $[8,25]$.

\section{HCV infection}

Hepatitis C (HCV) infection usually shows a variable evolution according to the immune response of the host $[26,27]$. Both innate and adaptive immunity are important in modulating active infection and the failure of the host's immunity results in the persistence of viral replication guided by cytokines imbalance $[26,27]$. TNF- $\alpha$ is involved in the pathogenesis of the disease, presenting a correlation with liver damage [28]. Recent evidence suggests that treatment failure could be due to the up-regulation of genes coding for TNF- $\alpha$, and in responders to INF treatment a reduction of TNF- $\alpha$ has been reported. This evidence suggests that anti-TNF- $\alpha$ could be helpful in patients with HCV infection [28]. A recent Italian multicentre cohort study assessed the long-term safety of adalimumab in a group of 17 psoriatic patients with chronic HBV infection and 20 psoriatic patients with chronic HCV infection [28]. All patients responded to treatment without re-activation of neither HBV nor HCV infection during a follow-up period of 27 and 40 months, respectively [29].

\section{Monitoring of patients during treatment with biological agents}

All patients need to be screened before starting treatment with biological drugs. Every 3 months patient's liver function tests should be performed. If HCV-Ab results are positive, HCV-RNA results are negative (HCV-Ab+, HCVRNA-), further monitoring is not necessary. If HCV-RNA is detectable, counselling with the ID (Infectious Disease) 
physician is needed to start antiviral treatment for HCV. Cirrhosis is a strict contraindication for biological treatments [30, 31].

\section{HIV infection}

The human immunodeficiency virus (HIV) causes a persistent, clinically silent, long-lasting chronic infection, resulting after a variable period (averaging 10 to 12 years) in the acquired immunodeficiency syndrome (AIDS) [32]. Psoriasis could be sometimes the first manifestation of viral infection [33-35] describing an immunological paradox: the activation of $T$ cells and treatments that reduce the $T$ cell count improve psoriasis, however when psoriasis is associated with HIV infection, it is more serious due to the low control of the immune response (with a T CD4 $+<200$ cell lymphocyte count per $\mu$ l, there is a risk 9 times greater to present a serious psoriasis form). TNF and its receptors represent an integral part of our immune system and at the same time, TNF signals have a significant impact on the replicative cycle of HIV. In turn, HIV-encoded proteins are able to modulate the TNF signalling pathway, resulting in the survival of HIVinfected cells and the death of "bystander cells", accidentally involved in the infectious process [34].

\section{Monitoring of patients during treatment with biological agents}

Guidelines for the management of psoriasis in HIV patients have been published in 2010 by the Medical Board of the National Psoriasis Foundation of the American Academy of Dermatology [36, 37].

As highlighted by the therapeutic algorithm of these guidelines, the management of HIV-related psoriasis includes antiretroviral therapy and concomitant anti-TNF- $\alpha$ drugs for the treatment of psoriasis with a positive effect on the immune reconstitution syndrome (IRIS) observed in these patients [36, 37].

Serological screening for HIV before initiating antiTNF- $\alpha$ therapy must be performed. It should also be performed in case of severe forms of psoriasis and/or in case of non-responders to therapies.

If the outcome of the HIV test is positive, an infectious diseases physician should be consulted to stage the infection by HIV-RNA levels and CD4 lymphocyte count. Anti-TNF therapy should not be administered if the CD4 count is less than 200 or if the patient is viremic for HIV.

The efficacy of anti-TNF- $\alpha$ in the treatment of seropositive patients is reported in the literature. For example, Hani Almoallim et al. reported three clinical cases regarding the use of anti-TNF- $\alpha$ in patients with psoriatic arthritis associated with HIV, two of them had coinfection by HBV and HCV [38]. Data show the safety of anti-TNF- $\alpha$ therapy in these patients: no one has shown an increase in viral load and/or a reduction in CD4 counts during treatment. Therapy safety is also maintained in the presence of HBV and HCV coinfection and concomitant diseases, such as compensated cirrhosis and kidney failure in dialysis treatment. In addition, in 2 patients, etanercept proved to be ineffective, so treatment with adalimumab was subsequently started [39].

Another report of an Italian 49-year-old patient with psoriatic arthritis and HIV shows a complete clinical resolution of skin manifestations and an improvement of joint symptoms during adalimumab treatment [39]. Interestingly, an increase in CD4 count and reduction in viral load has been documented [39].

\section{Discussion}

According to literature analysis, we provide a specific screening and management for infectious diseases in patients with moderate to severe plaque psoriasis, as mentioned above.

\section{Baseline screening (TO)}

Tuberculosis (TB): patient history (immunosuppression, occupation, origin from high-risk endemic areas, previous TB episode, other risk factors for TB, BCG vaccination, previous RX chest), IGRA (Quantiferon), RX chest (if doubtful, also CT scan) \pm Mantoux test (only if not vaccinated with BCG); other clinical and microbiological findings are necessary in case of a suspected active TB.

- In case of active TB (confirmed or suspected), biological therapy should be administered and an infectious diseases physician should be consulted.

In case of latent TB or suspected latent TB (Quantiferon +, visible lesions at RX chest even if Quantiferon is negative, with an already excluded active TB), isoniazid (300 mg/daily) administration starting 1 month before the beginning of biologic therapy, the therapy must be continued for 6 months. Liver function tests should be performed every month and $\mathrm{B}_{6}$ vitamin should be administered for the whole treatment duration.

\section{HBV}

All patients should be screened by performing: HBsAg, HBc-Abs (lgG), HBs-Abs, liver function tests.

- In case of HBsAg+: an ID specialist should be consulted to evaluate if the patient represents an active or inactive carrier. HBV-DNA, quantitative HBsAg, HBeAg, HBeAb (IgG) should be measured. Vaccination for hepatitis $A$ is recommended.

- If HBV-DNA is detectable: viral genotyping and treatment with entecavir or tenofovir should be started before biological treatment. Biological treatment should be initiated when HBV DNA is negative (or HBV DNA $<2000 \mathrm{UI} / \mathrm{ml}$ ). Antiviral treatment should be continued during biological therapy and not be discontinued even in case of a biological therapy interruption. The infectious diseases physician should be consulted. 
- In case of HBsAg+ and if HBV-DNA is not detectable: treatment with lamivudine is necessary (100 mg/daily) 2 weeks before starting and up to the end of biological therapy. In this case, lamivudine should be continued for 6 months after treatment discontinuation.

- In case of HBsAg-, HBcAb+ (HBsAb+ and HbsAb-): prophylaxis is not required, but clinical and laboratory monitoring is recommended.

- In case of HBsAg-, HBc-Abs-, HBs-Abs- in an unvaccinated patient or a vaccinated patient but with lost immunological coverage: vaccination before biological treatment is recommended. Any refusal of vaccination by the patient does not preclude the administration of these agents.

- In case of HBsAg-, HBc-Abs-, HBs-Abs+ > $10 \mathrm{UI}$ in a vaccinated patient still covered by immunization: no measure is required.

- In case of HBsAg-, HBc-Abs-, HBs-Abs+ < $10 \mathrm{UI}$ in a vaccinated but not completely protected patient: vaccination is recommended, however any refusal of the patient does not preclude the administration of biological therapies.

\section{$\mathrm{HCV}$}

All the patients should be monitored as for AST, ALT, HCV-Abs.

- In case of HCV-Abs+: HCV-RNA should be monitored.

- In case of HCV-RNA+: liver function tests are indicated and Fibroscan could be performed when it is possible.

In order to begin the anti-HCV treatment, an ID specialist should be consulted. If antiviral therapy is not started due to contraindication or for other reasons, therapy with biologics can be started anyway. Biological drugs should not be prescribed if cirrhosis is not controlled due to the higher infective risk.

\section{HIV}

Before starting biological treatment HIVAb lab test should be performed. If HIV test results are positive, it is necessary consult an ID physician to decide whether to administer biological treatment.

- In case of HIV+: viral load (HIV-RNA) and CD4+ count should be measured.

- Biological drugs should not be administered if CD4+ count is $<200 / \mu l$.

\section{Conclusions}

All psoriatic patients being candidates to start a biological therapy should be subjected to careful screening to reduce the infectious risk. Furthermore, it is recommended to follow an appropriate therapeutic management in psoriatic patients affected by infectious comorbidities in therapy with biological drugs. In these cases, close collaboration with the infectious diseases physician is essential, thus an improvement in multidisciplinary approach is desirable all over Italy [40].

\section{Conflict of interest}

The authors declare no conflict of interest.

\section{References}

1. Nestle FO, Kaplan DH, Barker J. Psoriasis. N Engl J Med 2009; 361: 496-509.

2. Parisi R, Symmons DP, Griffiths CE, Ashcroft DM. Global epidemiology of psoriasis: a systematic review of incidence and prevalence. J Invest Dermatol 2013; 133: 377-85.

3. Hwang ST, Nijsten T, Elder JT. Recent highlights in psoriasis research. J Invest Dermatol 2017; 137: 550-6.

4. Jiaravuthisan MM, Sasseville D, Vender RB, et al. Psoriasis of the nail: anatomy, pathology, clinical presentation, and a review of the literature on therapy. J Am Acad Dermatol 2007; 57: 1-27.

5. McGonagle D. Enthesitis: an autoinflammatory lesion linking nail and joint involvement in psoriatic disease. J Eur Acad Dermatol Venereol 2009; 23 Suppl 1: 9-13.

6. Richard MA, Barnetche T, Rouzaud M, et al. Evidence-based recommendations on the role of dermatologists in the diagnosis and management of psoriatic arthritis: systematic review and expert opinion. J Eur Acad Dermatol Venereol 2014; 28 Suppl 5: 3-12.

7. Kasiraman V, Atwan AA, Durojaiye OC, et al. Risk of tubercolosis with the use of anti-TNF medications in psoriasis: incidence, screening and management. Dermatol Online J 2014; 20: 13030/qt8s09h73j.

8. Bonifati C, Lora V, Graceffa D, Nosotti L. Management of psoriasis patients with hepatitis $B$ or hepatitis $C$ virus infection. World J Gastroenterol 2016; 22: 6444-55.

9. Kumar A, Abbas W, Herbein G. TNF and TNF receptor superfamily members in HIV infection: new cellular targets for therapy? Mediators Inflam 2013; 2013: 484378.

10. Pai M, Behr MA, Dowdy D, et al. Tuberculosis. Nat Rev Dis Primers 2016; 2: 16076.

11. Pescitelli L, Ricceri F, Prignano F. Tuberculosis reactivation risk in dermatology. J Rheumatol Suppl 2014; 91: 65-70.

12. Cantini F, Nannini C, Niccoli L et al.; SAFEBIO (Italian multidisciplinary task force for screening of tuberculosis before and during biologic therapy). Guidance for the management of patients with latent tuberculosis infection requiring biologic therapy in rheumatology and dermatology clinical practice. Autoimmun Rev 2015; 14: 503-9.

13. Amerio P, Amoruso G, Bardazzi F, et al. Detection and management of latent tuberculosis infections before biologic therapy for psoriasis. J Dermatolog Treat 2013; 24: 305-11.

14. Sauzullo I, Mengoni F, Marocco R, et al. Interferon-gamma release assay for tuberculosis in patients with psoriasis treated with tumour necrosis factor antagonists: in vivo and in vitro analysis. Br J Dermatol 2013; 169: 1133-40.

15. Pai M, Denkinger CM, Kik SV, et al. Gamma interferon release assays for detection of Mycobacterium tuberculosis infection. Clin Microbiol Rev 2014; 27: 3-20.

16. Drago L, Nicola L, Signori V, et al. Dynamic QuantiFERON response in psoriasis patients taking long-term biologic therapy. Dermatol Ther (Heidelb) 2013; 3: 73-81.

17. Scrivo R, Sauzullo I, Mengoni F, et al. The role of interferongamma release assays in predicting the emergence of active tuberculosis in the setting of biological treatment: a case 
report and review of the literature. Clin Rheumatol 2016; 35: 1383-8.

18. Scrivo R, Sauzullo I, Mengoni F, et al. Mycobacterial interferon-gamma release variations during long term treatment with tumor necrosis factor blockers: lack of correlation with clinical outcome. J Rheumatol 2013; 40: 157-65.

19. Pai M, Behr M. Latent Mycobacterium tuberculosis infection and interferon-gamma release assays. Microbiol Spectr 2016; 4: doi: 10.1128/microbiolspec.TBTB2-0023-2016.

20. Ott JJ, Horn J, Krause G, Mikolajczyk RT. Time trends of chronic HBV infection over prior decades - a global analysis. J Hepatol 2017; 66: 48-54.

21. Gish RG, Given BD, Lai CL, et al. Chronic hepatitis B: virology, natural history, current management and a glimpse at future opportunities. Antiviral Res 2015; 121: 47-58.

22. Viganò M, Serra G, Casella G, et al. Reactivation of hepatitis $B$ virus during targeted therapies for cancer and immunemediated disorders. Expert Opin Biol Ther 2016; 16: 917-26.

23. Mastroianni CM, Lichtner M, Citton R, et al. Current trends in management of hepatitis $B$ virus reactivation in the biologic therapy era. World J Gastroenterol 2011; 17: 3881-7.

24. Viganò $M$, Degasperi $E$, Aghemo A, et al. Anti-TNF drugs in patients with hepatitis B or C virus infection: safety and clinical management. Expert Opin Biol Ther 2012; 12: 193-207.

25. Prignano F, Tripo L, Amato L, et al. Tuscan consensus on the diagnosis, treatment and follow-up of moderate-to-severe psoriasis. G Ital DermatolVenereol 2017; 152: 99-108.

26. Thrift AP, El-Serag HB, Kanwal F. Global epidemiology and burden of HCV infection and HCV-related disease. Nat Rev Gastroenterol Hepatol 2017; 14: 122-32.

27.Zuniga El, Macal M, Lewis GM, Harker JA. Innate and adaptive immune regulation during chronic viral infections. Annu Rev Virol 2015; 2: 573-97.

28. Nelson DR, Lim HL, Marousis CG, et al. Activation of tumor necrosis factor-alpha system in chronic hepatitis C virus infection. Dig Dis Sci 1997; 42: 2487-94.

29. Piaserico S, Dapavo P, Conti A, et al. Adalimumab is a safe option for psoriasis patients with concomitant hepatitis B or C infection: a multicentre cohort study of 37 patients and review of the literature. J Eur Acad Dermatol Venereol 2017; 31: 1853-9.

30. Brunasso AM Puntoni M, Giulia A, Massone C. Safety of antitumour necrosis factor agents in patients with chronic hepatitis C infection: a systematic review. Rheumatology 2011; 50: 1700-11.

31. Caso F, Cantarini L, Morisco F, at al. Current evidence in the field of the management with TNF-alpha inhibitors in psoriatic arthritis and concomitant hepatitis $\mathrm{C}$ virus infection. Expert Opin Biol Ther 2015; 15: 641-50.

32. Deeks SG, Lewin SR, Havlir DV. The end of AIDS: HIV infection as a chronic disease. Lancet 2013; 382: 1525-33.

33. Deeks SG, Tracy R, Douek DC. Systemic effects of inflammation on health during chronic HIV infection. Immunity 2013; 39: 633-45.

34. Morar N, Willis-Owen SA. Maurer T, Bunker CB. HIV-associated psoriasis: pathogenesis, clinical features, and management. Lancet Infect Dis 2010; 10: 470-8.

35. Gallitano SM, McDermott L, Brar K, Lowenstein E. Use of tumor necrosis factor (TNF) inhibitors in patients with HIV/ AIDS. J Am Acad Dermatol 2016; 74: 974-80.

36. Patel RV, Weinberg JM. Psoriasis in the patient with human immunodeficiency virus. Part 1: review of pathogenesis. Cutis 2008; 82: 117-22.
37. Menon K, Van Voorhees AS, Bebo BF Jr, et al.; National Psoriasis Foundation. Psoriasis in patients with HIV infection: from the medical board of the National Psoriasis Foundation. J Am Acad Dermatol 2010; 62: 291-9.

38. Almoallim H, Jali I, Wali G. Successful use of antitumor necrosis factor-alpha biological therapy in managing human immunodeficiency virus-associated arthritis: three case studies from Saudi Arabia. Joint Bone Spine 2013; 80: 426-8.

39. Lindsey SF, Weiss J, Lee ES, Romanelli P. Treatment of severe psoriasis and psoriatic arthritis with adalimumab in HIVpositive patient. J Drugs Dermatol 2014; 13: 869-71.

40.Gisondi P, Cazzaniga S, Chimenti S, et al.; Psocare Study Group. Latent tuberculosis infection in patients with chronic plaque psoriasis: evidence from the Italian Psocare Registry. Br J Dermatol 2015; 172: 1613-20. 